\title{
Energy and the environment
}

\author{
O. T. Inal, P. F. Gerity \& D. D. H. López \\ New Mexico Institute of Mining and Technology, USA
}

\begin{abstract}
Energy is one of the major ingredients required for human sustenance and comfort. Yet, energy has an environmental impact at every stage of its acquisition as well as its use. We produce significant environmental waste and impact extracting energy, transporting the extracted material(s), and in processing it to render it useable. The global warming gases generated and the associated effects represent only a small sample of the serious by-products of our energy use, and only a fraction of the devastation that we presently bestow on our planet.

Presently, we are all aware that the price of one barrel of crude oil has exceeded the $\$ 75.00$ level for the first time in history, and the negative impact on our individual economics as well as that affecting the industrialized world is such that every segment of our quality of life and living standard is threatened. These increased costs do not produce any direct benefit to the planet, but only contribute to the total global decline of living conditions and standards. The actual monetary benefit is only enjoyed by a very few as well.

Currently, the only fuel source alternative which seems to be considered politically acceptable involves the conversion of biomass (such as maize, sugar cane, other vegetable sources or use[s]) into bio-fuels to partially replace the shrinking supply of traditional sources of hydrocarbons. However, there is a finite and limited amount of arable land that can efficiently produce and successfully harvest biomass. All human needs for food and fiber must compete for access to this land area with any proposed use for energy production. The competition among food, fiber and fuel is already very stressed, and the use of bio-mass is not a viable, long-term solution to the problem.

This paper identifies fuel sources, the quantities currently available, and projections for the future. The areas of solar, wind, hydrogen, and renewed interest in nuclear power are all considered. It is our effort to put these alternative options into a more realistic light.

Keywords: energy, biomass, biodiversity, pollution, fusion, fission.
\end{abstract}




\section{Introduction}

For many, the state of the world is getting worse based on four major fears which they harbor. These four fears include:

\subsection{Natural resources are running out}

Their quantities are not of such an extent that their production could cope with the needs of a more industrialized and expanding population. Since the next million or so years will only net an accumulation of about one six hundredths of the amount built up thus far, it cannot be relied upon to replenish itself.

Although the amounts of these resources are limited, the limits are far greater than many project. The "main three" sources and the most recent assumed quantities are [1]:

1. Coal Reserves: Enormous; greater than 2000 years' supply

2. Natural Gas: (in trillion cubic feet)

\begin{tabular}{|l|l|l|l|l|l|}
\hline Total & $\begin{array}{l}\text { Middle } \\
\text { East }\end{array}$ & Russia & $\begin{array}{l}\text { North } \\
\text { America }\end{array}$ & $\begin{array}{l}\text { US } \\
\text { Reserves } \\
(1999)\end{array}$ & $\begin{array}{l}\text { US } \\
\text { Reserves } \\
(2000)\end{array}$ \\
\hline 6000 & 2500 & 1600 & 269 & 167 & 189 \\
\hline
\end{tabular}

US Annual Usage: 18-20, i.e. less than ten year domestic year supply, but reserve growth is outpacing depletion at the moment.

3. Producible petroleum reserves (Billions of barrels)

Conventional:

World $\quad 1,300$

Middle East $\quad 700$

North America 40

Tar Sands:

Canada alone $\quad 175$

Oil Shale:

Recoverable today 200

Annual Usage:

$\begin{array}{lr}\text { USA } & 7 \\ \text { World } & 30\end{array}$

There is greater than a 50 year supply at current usage rate. (It also puts into question as to why we have a sudden increase in per barrel crude oil prices).

The projected recoverable reserve today seems to be:

$\begin{array}{llr}\text { Estimate } & \text { Quads } & \text { Years Supply } \\ \text { Fossil Fuels } & 200,000 & 600 \\ \text { Nuclear } & 430,000 & 1400 \\ \text { Geothermal Fluids } & 160,000 & 500\end{array}$

i.e. no urgent cessation of energy delivery from any of these supplies at the present time. The environmental cost though is large. 


\subsection{Population is ever growing, leaving less and less to eat}

Agricultural production in the developing world has increased by $50 \%$ per person since 1961 (as of 2001). The food intake in poor countries has shown the following increases: 1,932 (1961), 2,650 (1998) and expected to be 3,620 (2030) Calories; i.e. not a lessening but a steady increase in nourishment.

In contrast, starvation in developing countries was $45 \%$ in 1949 has reduced to $18 \%$ in 2001 and is expected to be $12 \%$ in 2010 [2]. Also, since year 1800 food prices have decreased by more than $90 \%$ and in year 2000 prices were lower than ever before [3]. It turns out, as people grow richer and healthier, they tend to have smaller families. While the population growth rate mentioned was greater than $2 \%$ per year in early 1960 's, it became $1.26 \%$ in year 2001 and the expected the rise will be $0.46 \%$ in year 2050 . UN estimates show that population growth will be over in year 2600 stabilizing at just 11 billion. It turns out that human ingenuity has boosted food production not merely in line with, but ahead of, population growth with a concomitant reduced pressure on land acquisition and thus biodiversity.

\subsection{Biodiversity loss}

With forced increase in arable land, and thus decrease in forested area, biodiversity is expected to suffer the greatest loss. This doesn't seem to be the case when one considers that the Eastern United States, that had its forested areas reduced to $1-2 \%$ of their original size, in the last two centuries, has resulted in the extinction of one forest bird. All but $12 \%$ of forested area being cleared in Brazil has lead to no verifiable extinction of any species. Further, UN reports that tropical forest losses in the last century is less than $0.5 \%$ rather than the 2$4 \%$ as specified by environmentalists.

\subsection{The Planet's air and water are becoming ever more polluted}

This is another area that is exaggerated. Although there is pollution related to every stage of energy use, this is far below what some project. Human endeavor is actually very small in comparison to natural activity on the planet and could only be the "last straw that broke the camel's back", as the saying goes. A volcano produces more $\mathrm{SO}_{\mathrm{x}}$ and $\mathrm{CO}_{2}$ than hundreds of industrial facilities. Air pollution is increasing in developing countries; they are merely duplicating the development route of the industrial ones. When they get to be economically stable they too will address industrial pollution.

\subsection{Why a disjunction between perception and reality?}

It is clear that a disjunction exits between what some are claiming and reality is. This possibly comes about, due to the following:

\subsubsection{Research in any given area initiates with the definition of the existence of a problem in that area}

This unfortunately leads to the impression that many more problems exist than is the case. 


\subsubsection{Activists need to be noticed}

To achieve this interest they tend to exaggerate considerably (one extremist environmental scientist wanted to destroy, with a nuclear bomb, humanity (and bring it back to the stone age) so that he, without polluting instruments of modern life, could live without sin [4]. A good example in this regard is the estimation of the worldwide fund (1977) that two thirds of world's forests were extinct; the truth is this number is nearer $20 \%$. Also there is the inherent suspicion of people; a trade organization is either altruistic - when it goes for stronger pollution controls - and is instantly seen as self-interested if it argues for weaker controls.

\subsubsection{People are clearly more curious about bad news than good}

Although 1997-1998 El Niño phenomena was blamed for damages amounting to $\$ 4$ billion, the $\$ 19$ billion benefits were generally not as widely reported.

\subsubsection{There is also the wrong perception about the environment}

After the infamous rubbish loaded barge made its travel through the Caribbean bringing refuse to unload at every port, unsuccessfully returning to its original port, New York, people began talking about the amount of waste created in the US. The actual case is that America would produce, through the entire $21^{\text {st }}$ century, enough waste that will take up an area $1 / 12^{\text {th }}$ of the country.

In this regard, ignorance matters only when it leads to faulty judgments; for example, cost of Kyoto, for US alone, will be higher ( $\$ 1$ trillion) than five times the money needed to cure a major problem for the planet: universal access to clean drinking water and sanitation. In this regard, it may be costly to be overly optimistic but more costly still to be too pessimistic.

\section{Energy options for the future}

As Sheik Yamani, Saudi Arabia's former oil minister and a founding member of OPEC, is said to have pointed out: "The stone age came to an end not for a lack of stones, and the oil age will end, but not for a lack of oil" [5]. Humanity stopped using stones because we found superior materials (iron and bronze) and likewise we probably will stop using oil when other energy technologies provide superior benefits. What we have said to this point is that it is not that we are going to run out of energy but that we are going to have it at a higher cost. The rate of our consumption of energy related materials has depended on human ingenuity in that when cereal consumption increased twofold since 1960, meat consumption increased by threefold since 1960, fish catch raised its take to six times between 1950 to 1997 this was balanced by the fact that half of the commercial fertilizer ever produced was applied to farming since 1984 [6]. Life on earth exists only because of the natural greenhouse effect, the ability of the atmosphere to retain enough heat for species to thrive (and no more).There is a finite amount of carbon on the planet: stored in fossil fuels, the sea, living matter and the atmosphere. Without human influence, transfers between these storage areas roughly balance each other; for example: plants take up carbon as they 
grow but release it as they decay. Humans cut down trees and burn fossil fuels and thus release extra carbon into the atmosphere increasing the greenhouse effect. The greenhouse gases include carbon dioxide, emitted by fossil fuel burning and deforestation, and methane, released from rice paddies, landfill sites and animal waste. Warming that comes about, from the greenhouse effect, will trigger some processes which speed further warming and other effects which mitigate it (decreased ice cover produce exposed land which leads to more absorption of heat and speeds warming further; in contrast, plants' $\mathrm{CO}_{2}$ intake is likely to increase as higher temperatures increase growth rates). A danger of increasing warming is to either alter or stop natural occurrences on the planet that equilibrate climate changes; for example, the "Great Ocean Conveyor", surface and deep sea movements, in the form of Gulf stream uses the wind driven surface currents and carry the milder tropic weather to Northern Europe and thus make life quite bearable [7].

The, so-called, "infinite" sources of power - sources that are continuously replenished - fall into two groups:

\subsection{Group A}

\subsubsection{Hydrogen}

Abundant and clean but must be processed for use as a fuel. The product of its use is water vapour. There is currently no economic way to dissociate water and thus we still have to develop an efficient means of doing this and to store and distribute it. Its use in transportation vehicles will have to be preceded by careful engineering of its containment due to its highly explosive characteristic.

\subsubsection{Wind}

Wind power can be obtained by careful design and with knowledge of the stream flow pattern(s) of air. Large scale use is very impractical. It is a good source for exposed areas, is environmentally friendly and is a relatively endless source. Noise of its operation and the obtrusiveness of its appearance are its major distractions. Few seem to understand the metal fatigue and mechanical failure which occurs. The likelihood of a windmill having extended life, and thus be cost effective, is, therefore, rather small.

\subsubsection{Water}

Safe and pollution free source of energy that is limited by location and by the upheaval it can cause since it requires big sites for collection. Water power is assumed to have a capacity of three trillion watts, which is equivalent to present total energy use in industry. Only $8.5 \%$ of this power though is utilized at present because of the lack of industrial development in regions such as South America, Africa, and Southeast Asia which are of highest potential for hydroelectric power.

The efficiency of use of these "infinite" sources is low and they have restrictions of locations, seasons and large capital investment is required [8]. 


\subsection{Group B alternatives}

\subsubsection{Solar energy}

Solar energy is derived from the fusion reactions that take place in the core of the sun. The main obstacle to date in efficiently harnessing this power has been finding ways or collectors to economically concentrate the available low energy density of the solar radiation. Also, the intermittent nature of solar energy requires an efficient storage capability in utilizing this source.

Collector designs are based on a thermal process in which the temperature of a working fluid, typically water or alcohol, is increased by absorbed solar radiation. Techniques utilized in collection of this energy include:

\subsubsection{Concentrating solar collectors}

A parabolic mirror system concentrates solar irradiation onto a pipe with a selective absorber coating placed at the focal point containing the heat transfer medium. Temperatures in excess of $500^{\circ} \mathrm{C}$ have been achieved with this design but high installation costs have limited their use at present. To attain highest efficiencies, a tracking system that follows the sun is needed. The collector tube assembly includes a one and a quarter inch stainless steel tubing, 12 feet long, that has a coat of conductor sulfamate nickel - and a double coat of absorber metal - black chrome or black nickel - to gain absorbtivity efficiencies above $95 \%$. In the actual design of this tubing the arrangement of the above layers are: stainless tube, nickel coating, black chrome or black nickel layer followed by a vacuum jacket and a glass cover to reflect back all reflections from the absorber surface. As can be imagined, this is extremely costly together with being an extremely bulky assembly.

\subsubsection{Flat plate collectors}

A cheaper version consisting of a black coating over a flat surface that is attached to copper tubing carrying the exchange fluid - usually water. The absorbed thermal energy by the black surface heats the exchange medium that flows in copper pipes to the storage facility. The idea in this process is to supplement the regular heating source with solar energy. This design is cheaper and has been incorporated into the construction of many houses already. According to a study done at Los Alamos National Laboratories, this design could produce up to $75 \%$ of the total energy needs of a household in a geographic region like New Mexico. Therefore, the main problem with this approach is the fact that, to achieve the power consumption of U.S. in 1970, the area of collection for solar irradiation would be approximately 24,500 square kilometers [9].

It is evident at this point that except for solar energy, all the other forms mentioned are not plentiful enough to supply the future demand of energy but can and should all be utilized as limited contributions to the total picture. More efficient collection and storage of solar energy can lead to an unlimited source of clean energy for future. 


\section{Nuclear energy: fusion and fission}

To understand the basic principles involved in Fission and Fusion, nuclei and the arrangement of neutrons and protons within these have to be studied. In a nuclear plant the energy is released from the nuclei of the atoms while energy derived from fossil fuel burning (coal, oil, or gas) comes from the atoms or molecules composed of atoms.

The binding energy for nucleons is much greater than the binding energy of atoms; for example helium atom with two protons has a binding energy per atom of $19 \mathrm{eV}$ (electron volts) while helium nucleus with two protons and two neutrons (four nucleons) has a binding energy of $7.0 \mathrm{MeV}$ (million electron volts) per nucleon.

Energy per nucleon goes through a minimum for elements with 50 to 130 nucleons rising sharply for lighter nuclei and gradually toward the heavy nucleus end. This indicates that it is possible to decrease internal energy and thereby release the excess energy by fusing lighter nuclei together and move towards the middle of this curve or splitting an unstable heavy nuclei into two intermediate nuclei and again release large quantities of energy. The former is the principle behind fusion and the latter is utilized in fission reactions. Although it might seem that more energy release occurs in fusion, this is energy per nucleon and the number of nucleons participating in fission is much more. Actual energy available from fusion is only about ten percent of the energy derivable from fission [10].

Nuclear breeding is achieved with the neutrons released by nuclear fission. The fissioning of each atom of a nuclear fuel, such as ${ }^{235} \mathrm{U}$, liberates an average of more than two fast (high energy) neutrons. One of the neutrons must trigger another fission to maintain the nuclear chain reaction and there is at least one neutron available to breed new fissionable atoms, that is, to transform "fertile" isotopes of heavy elements into fissionable isotopes. This is illustrated as follows [11-13].

Uranium Cycle:

2. ${ }_{92}^{238} U_{146}+\mathrm{n} \rightarrow{ }_{92}^{239} U_{147} \rightarrow{ }_{93}^{239} N p_{146} \rightarrow{ }_{94}^{239} P u_{145}$

and, Thorium cycle:

$$
\text { 3. }{ }_{290}^{232} \mathrm{Th}+\mathrm{n} \rightarrow{ }_{290}^{233} \mathrm{Th} \rightarrow{ }_{291}^{232} \mathrm{~Pa} \rightarrow{ }_{92}^{233} \mathrm{U}
$$

The two man-made isotopes ${ }^{239} \mathrm{Pu}$ and ${ }^{233} \mathrm{Pu}$ are fissionable like ${ }^{235} \mathrm{U}$ and this way the energy source material is increased tremendously.

The reactor core assembly, consists of fuel elements (clad in thin high melting point, high thermal conductivity metal containers), control rods and the cooling medium surrounding the assembly.

When cooling is done with water, dual purpose is served. Water removes the heat of fission from the containers and also acts as a moderator. To slow down the neutron of $1 \mathrm{MeV}$ energy to less than the $1 \mathrm{eV}$ is accomplished by some 20 
or so collisions before it is absorbed by a ${ }^{235} \mathrm{U}$ and induce fission. Control rods used in the core area regulate the neutron flow. The heat carried away by the cooling media is converted to electricity through a generator.

\section{Environmental considerations}

To summarize, with a power system based on neutrons the following environmental advantages are attainable:

- With the fusion and/or fission reactors no burning of the world's oxygen or hydrocarbon resources and hence no release of carbon dioxide or other combustion products are brought about for power generation.

- Fusion creates no wastes and the high energy neutrons can be utilized to burn radioactive wastes of the fission reactors.

- Fusion reactors are also inherently incapable of a "run away" accident, there is never enough fuel present at any one time to support a nuclear excursion.

These concepts are only concepts and years of research based on the most advanced technologies are required to make these reactors operate with efficiency and be failsafe. The increasing demand for energy and decreasing amount of fossil fuels have today made the topic of nuclear reactors very prominent, since this is the only energy form in magnitude and long range usability that is at hand.

\section{$5 \quad$ Fuelish?}

A logical conclusion of this study would be to question the merits of a spiraling increase of our energy needs. Since there are no "pure" forms of energy with no dangers associated or no pollution resulting there from, shouldn't the goal be to curb this energy hunger and go for a zero-based population growth rather than finding new ways of satisfying this increasing need? The prospects we are faced with if the present trend continues are as follows.

\subsection{If the source is coal}

- It would require 33 tons of coal/person/year; which we might be able to pay for but the environmental cost might be too large.

- Would result in 20 trillion tons of $\mathrm{CO}_{2}$ emitted into the earth's atmosphere which could involve enough atmospheric changes that melting of the polar caps would become reality. The excavations for coal would leave the countryside an environmental mess and thousands of vehicles required to transport the coal could congest traffic in every major city. 


\subsection{If the source is nuclear}

- $\quad$ Fissionable U-235 is of very limited quantity and would only last about fifty years.

- Breeder reactor that would have to take the slack-breeding ${ }^{238} \mathrm{U}$ and ${ }^{232} \mathrm{Th}$ into ${ }^{234} \mathrm{Pu}$ and ${ }^{233} \mathrm{U}$ - are the worst offenders in thermal pollution. The product elements are also the major components of nuclear weapons.

- 11,000 megacuries of long-lived radioactive isotopes that would be produced each year would have to be transported between the reactor site and the processing plants - where they are made into bricks - and ultimately stored at a disposal site.

This leads to only one conclusion and that is to control energy consumption and population explosion together since there are no "absolutely clean" forms of energy production.

\section{References}

[1] Oil and Gas Journal, Jan 1, 2005.

[2] B. Lomborg, The Economist, Aug 4, (2001), 63-65.

[3] World Bank, 2001.

[4] Dixy Lee Ray with L. Guzzo, "Trashing the Planet", Regnery Gateway, Washington D.C. 1987.

[5] Greider, William 2000 “Oil on Political Waters”. Nation, 10/23/2000 271 (12):5-6.

[6] Alex Kirby, http://newsuote.bc.co.uk/mpapps/pagetools/print/news.bbc/ co.us/z/.

[7] "Guide to Climate Change", http://news.bbc.co.uk/z/shared/spl/hi/scinat $/ 04 /$ climate change $/ \mathrm{html} / \mathrm{climate} . \mathrm{stm}$

[8] M. King Hubert. Scientific American, 224, \#3, Sept(1971) 60.

[9] M. King Hubert. Scientific American, 224, \#3, Sept(1971) 62.

[10] D.R. Inglis, Nuclear Energy, Its Physics and its Social Challenge, Addison Wesley, Pub. Co. Reading, Mass (1973).

[11] G.I. Scaborg and J.L. Bloom, Scientific American, Vol. 223, No.5, No. (1970) 13.

[12] The Complete Ecology Handbook; Philip Nobile and John Dealy, Eds., Doubleday and Co. Inc, NY (1972).

[13] W.H. Jordan, Physics Today, 23, \#5, May (1970) 32. 